\title{
Validity of Mycobacterium tuberculosis Antigen Cocktail: ESAT-6, CFP- 10, and MPT64 in Sputum and Cerebrospinal Fluid for Pulmonary Tuberculosis and Tuberculous Meningitis Diagnosis
}

\author{
Dewi Kartika Turbawaty, Nenny Gustiani, Livia Noviani, Ida Parwati
}

Department of Clinical Pathology, Faculty of Medicine, Universitas Padjadjaran-Dr. Hasan Sadikin General Hospital

\begin{abstract}
Objective: To determine the validity of tuberculosis (TB) antigen cocktail (ESAT-6, CFP-10 and MPT64) for pulmonary tuberculosis and TB meningitis diagnosis.

Methods: This is a descriptive observational study design. The study was conducted at the Clinical Pathology Laboratory of Dr. Hasan Sadikin General Hospital during September 2012 until March 2013 for the pulmonary tuberculosis study and from January 2014 to May 2014 for the TB meningitis study. The TB antigen cocktail rapid immunochromatography (ICT) test was conduncted on all samples. The sputum and cerebrospinal fluid (CSF) were cultured as gold standards.

Results: There were 149 pulmonary and 41 TB meningitis subjects. The sensitivity of rapid ICT TB antigen cocktail for diagnosing pulmonary tuberculosis was $95.7 \%$ with a specificity of $87.2 \%$. Of 41 TB meningitis subjects, based on Marais criteria, there were $6(16 \%)$ subjects with a definite TB meningitis, $26(63 \%)$ subjects with probable TB meningitis, and $9(21 \%)$ subjects with possible TB meningitis. The sensitivity and specificity of TB antigen cocktail rapid ICT for TB meningitis diagnosis were $83.3 \%$ and $68.5 \%$, respectively.

Conclusions: In this study, rapid ICT TB antigen cocktail (ESAT-6, CFP-10 and MPT64) from sputum sample has good validity for diagnosing a pulmonary

Received:

May 6, 2015 tuberculosis. Cerebrospinal fluid sample has moderate validity to diagnose TB meningitis.
\end{abstract}

Revised:

July 3, 2015

Keywords: $M$. tuberculosis culture, pulmonary TB, TB meningitis, TB antigen cocktail (ESAT-6, CFP-10 and MPT64) rapid ICT

Accepted:

August 28, 2015

IJIHS. 2015;3(2):60-5

\section{Introduction}

Tuberculosis (TB) remains a major public health problem in most developing countries. One-third of the world population carries an asymptomatic infection with Mycobacterium tuberculosis, resulting in eight million new cases of TB and two million deaths every year. ${ }^{1}$ Until now, the diagnosis of active tuberculosis is based on microscopy identification for acid-fast bacillus (AFB) and culture of $M$. tuberculosis. However, microscopy technique is

\section{Correspondence:}

Dewi Kartika Turbawaty, Department of Clinical

Pathology, Faculty of Medicine, Universitas Padjadjaran-

Dr. Hasan Sadikin General Hospital

Jl. Pasteur No. 38, Bandung, Indonesia

e-mail: dewikt_2006@yahoo.co.id insensitive and conventional culture, although it is more sensitive, is time consuming and requires safety precautions while during the long and tedious culture process an infected individual continues to spread the disease to many other susceptible individuals. ${ }^{2}$ The polymerase chain reaction (PCR) method reduces the time detection for culture and identification but requires trained persons, sophisticated equipment, and sophisticated techniques. Rapid and accurate TB diagnostics play an important role in detecting the disease. Currently, antigen secreted by M. tuberculosis (ESAT-6, CFP-10 and MPT64) encoded by the genes of "Region of Difference" (RD)1, RD2 and RD3 gives an opportunity for rapid TB diagnostic. ${ }^{3,4}$ 
M. tuberculosis genome consists of 16 regions of differences (RD). Among these regions, the RD1 locus, which plays a key role in the virulence of $M$. tuberculosis, presents in clinically pathogenic strains of $M$. tuberculosis and $M$. bovis, but is deleted in M. bovis BCG (bacillus Calmette-Guerin) vaccine strains and absent in mycobacteria other than $M$. tuberculosis (MOTT). ${ }^{5}$ This region contains nine protein coding genes (Rv3871-3879c), which probably encodes protective and/or virulent antigen. Among the major antigen of RD1 locus, early secretory antigenic target- 6 (ESAT-6) and culture filtrate protein-10 (CFP10) are encoded by Rv3875 and Rv3874 genes respectively, form a 1:1 heterodimeric complex in vitro. ${ }^{6-8}$ Those proteins are important antigen for IFN- $y$ producing T-cells active in the first phase of tuberculosis infection and their putative role in deactivation of the macrophage and dendritic cell functions. ${ }^{6}$ Tuberculosis MPT64, also termed as protein Rv1980c, is a protein secreted by actively growing $M$. tuberculosis strains. It has also been proven that MPT64 antigen is found only in viable and actively dividing cells of M. tuberculosis. ${ }^{9}$ Subjects of this study were pulmonary tuberculosis and TB meningitis because pulmonary tuberculosis account for $85 \%$ of all TB cases while TB meningitis was the most severe TB manifestation..$^{10,11}$

The aim of this study was to determine the validity of TB antigen cocktail (ESAT-6, CFP10 and MPT64) for the diagnosis of pulmonary tuberculosis and TB meningitis.

\section{Methods}

This study involved two groups of tuberculosis (TB) patients. The first group were patients diagnosed with pulmonary TB according to the International Standard of Tuberculosis (ISTC) and TB National Program. This study included new patients aged $>14$ years who presented at the outpatient TB DOTS clinic of Dr. Hasan Sadikin General Hospital, Bandung, Indonesia.

The second group was adult patients presented with suspected meningitis at Dr. Hasan Sadikin General Hospital. A clinical diagnosis of meningitis in this setting was based on Marais criteria (score $\geq 6$ ): definite TB meningitis was defined as cerebrospinal fluid (CSF) microscopy or positive culture for M. tuberculosis, Probable TB meningitis was defined if Marais score were 6-9 (without CT-scan) or 6-11 (with CT-scan) and possible TB meningitis was defined if Marais score were $\geq 10$ (without CT-scan) or $\geq 12$ (with CTscan). ${ }^{12}$ The exclusion criteria included the contaminated culture, traumatic CSF, patient who received anti TB agents $>7$ days, or patient who stopped TB treatment $\leq 14$ days.

This study was a cross-sectional descriptive observational study. The sputum specimens from the first group were obtained for direct examination of acid-fast bacilli (AFB) by ZiehlNeelsen (ZN) staining and cultured on Ogawa medium as gold standard. The niacin test was performed on all positive cultures to identify M. tuberculosis. The second group was a part of a project called 'Optimization of diagnosis of meningitis'. Following a lumbar puncture, 5-10 mL CSF was obtained, transported to the laboratory within one hour, and divided into two tubes: one $(2 \mathrm{~mL})$ for CSF cells, protein and glucose, one (7-8 $\mathrm{mL}$ ) for microbiological testing. The second tube was then concentrated by centrifugation at 3,000 g for 10 minutes. The CSF sediment was used to prepare smears for direct examination of acid-fast bacilli after Ziehl-Neelsen (ZN), Gram, and India ink stainings. Mycobacterial culture was used for
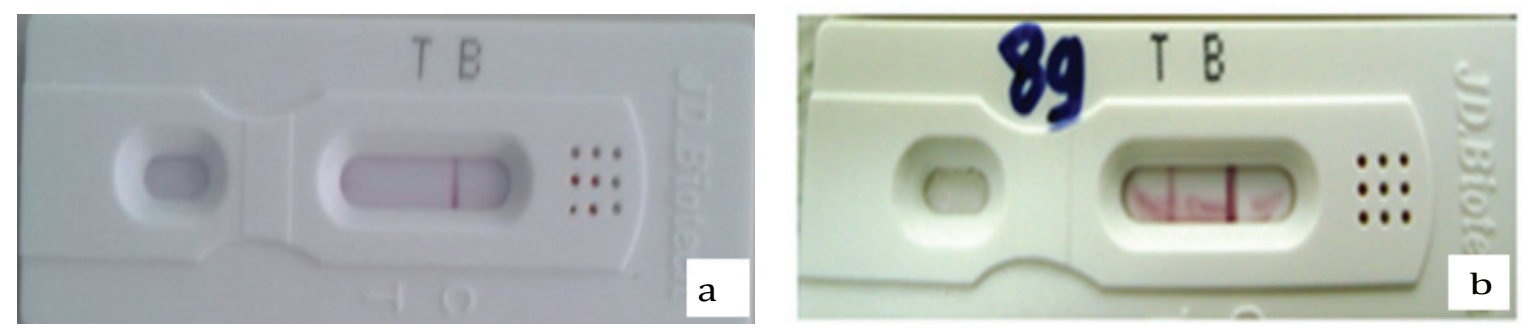

Fig. 1 Negative Rapid ICT TB Test Result (a), Positive Rapid ICT TB Test Result (b) 
Validity of Mycobacterium tuberculosis Antigen Cocktail: ESAT-6, CFP-10, and MPT64 in Sputum and Cerebrospinal Fluid for Pulmonary Tuberculosis and Tuberculous Meningitis Diagnosis

microscopic observation drug susceptibility (MODS). The TB antigen cocktail rapid ICT was performed on all samples by following the manufacturer's instructions (Jei Daniel Biotech Corp., Taiwan). One spot (500 uL) sputum or $100 \mu \mathrm{L}$ homogenize CSF specimen was submitted for examination, taking $1.5 \mathrm{~mL}$ of sample buffer into the specimen collection box. It was then mixed well with specimen by dropper up to down in 30-60 seconds then to stand for 30 minutes. This was pipetted to $100 \mathrm{uL}$ of supernatant sample and $100 \mathrm{uL}$ of sample buffer was added to the tube and mixed well for testing. Three drops (60 uL) were applied into " $\mathrm{S}$ " region of card for testing. The test result was read after 15 minutes. The result was considered positive if in addition to a pink colored control band, a distinct pink colored band also appear in test region. It was considered negative if only one colored band appears on the control region with no apparent band on the test region. The test result was considered invalid when there was a total absence of color in both regions, indicating procedure errors and/or test reagent deterioration (Fig. 1). The study was conducted at the Clinical Pathology Laboratory of Dr. Hasan Sadikin General Hospital.

ESAT-6, CFP-10 and MPT64 in sputum of patients with pulmonary tuberculosis and cerebrospinal fluid of patients with TB meningitis were using sensitivity, specificity, positive predictive value and negative predictive value. The positivity rate of TB antigen cocktail rapid ICT, microscopy and mycobacterial culture was expressed as percentage. Differences between groups of TB meningitis were compared using Fisher Exact test because had small sample size, with p-values $<0.05$ was considered statistically significant.
This study was approved by the Ethical Committee of the Faculty of Medicine, Universitas Padjadjaran-Dr. Hasan Sadikin General Hospital, Bandung, Indonesia. Written informed consent was obtained from either the patient or closest relatives (husband/wife or parents) for those who were unstable or unconscious at the time of presentation.

\section{Results}

From September 2012 to March 2013, there were 149 pulmonary tuberculosis subjects. Sputum culture was positive in $56(37 \%)$ samples, 86 (58\%) samples were negative and 7 (5\%) samples had contaminated culture. All contaminated samples were excluded. The niacin test was performed to all of 56 (37\%) positive culture samples and positive results were found in 49 (87.5\%); thus, the cultures were confirmed to be $M$. tuberculosis. There were $7(12.5 \%)$ cultures that were negative in niacin test and was confirmed to be MOTT. Tuberculosis antigen cocktail rapid ICT was positive in $58(40.8 \%)$ sputum samples, while all $7(100 \%)$ sputum samples from MOTT cultures had negative result. Thus, the sensitivity of sputum TB antigen cocktail rapid ICT was $95.7 \%$ and the specificity was $87.2 \%$ with a positive predictive value (PPV) of $81.0 \%$ and a negative predictive value (NPV) of $97.6 \%$ (Table 1 ).

From January 2014 until May 2014 there were 41 TB meningitis subjects, $6(16 \%)$ subjects with a definite diagnosis of TB meningitis base on Marais criteria, 26 (63\%) subjects with probable TB meningitis and 9 $(21 \%)$ subjects with possible TB meningitis. Using MODS culture as the gold standard, CSF TB antigen cocktail rapid ICT had a sensitivity

Table 1 Validity of TB Antigens Cocktail Rapid ICT from Sputum Specimens by M. tuberculosis Culture

\begin{tabular}{lcccc}
\hline & & \multicolumn{3}{c}{ M. tuberculosis cultured on Ogawa } \\
& & Positive & Negative & Total \\
\cline { 3 - 5 } TB Antigen Cocktail & Positive & 47 & 11 & 58 \\
& Negative & 2 & 82 & 84 \\
& Total & 49 & 93 & 142 \\
\hline
\end{tabular}

The sensitivity was $95.7 \%$ and the specificity was $87.2 \%$ 
Table 2 Validity of TB Antigen Cocktail Rapid ICT from Cerebrospinal Fluid (CSF) Specimens by M. tuberculosis Culture

\begin{tabular}{lcccc}
\hline & & \multicolumn{3}{c}{ MODS Cultured } \\
& & Positive & Negative & Total \\
\cline { 3 - 5 } TB Antigen Cocktail & Positive & 5 & 11 & 16 \\
& Negative & 1 & 24 & 25 \\
& Total & 6 & 35 & 41 \\
\hline
\end{tabular}

The sensitivity was $83.3 \%$ and the specificity was $68.5 \%$, PPV was $31.2 \%$, and NPV was $96 \%$

of $83.3 \%$ and the specificity was $68.5 \%$ with a PPV of $31.2 \%$, and a NPV of $96 \%$ (Table 2).

The positivity rate of TB antigen cocktail rapid ICT sputum was 40.8\% (58/142), AFB microscopy was $39.6 \% \quad(59 / 149)$ and Cultured on Ogawa from sputum specimens was 37.6\% (56/149) (Table 3).

The positivity rate of TB antigen cocktail rapid ICT, AFB microscopy and cultured on MODS from CSF specimens were 39\% (16/41), $5 \%(2 / 41)$ and $15 \%(6 / 41)$, respectively (Table 3). Based on Marais criteria, TB antigen cocktail rapid ICT was positive in 5 $(83 \%)$ definite patients, in $9(35 \%)$ probable patients and in $2(22 \%)$ possible patients. The positivity rate of TB antigen cocktail rapid ICT was higher in definite patients than in other groups $(\mathrm{p}=0.044)$.

\section{Discussion}

Tuberculosis is a chronic and endemic disease in Indonesia. Untreated or delay in treatment is associated with increased mortality and morbidity. Early laboratory diagnosis from clinical specimens is vital in the management of tuberculosis patients. Novel technologies for rapid detection of the disease has become a top priority not only in TB Research but also for diagnosis and treatment purposes. ${ }^{13}$ This study used TB antigen cocktail ESAT-6, CFP-10 and MPT64 because a combination of these proteins improves the sensitivity and specificity. $^{14}$

In this study, compared with Ogawa culture as a gold standard, TB antigen cocktail rapid ICT sputum had a sensitivity of $95.7 \%$ and specificity of $87.2 \%$. There were 11 culturenegative but TB cocktail antigen rapid ICT positive in those that had been taking fluoroquinolon drug for some days before, which may reduce the viability of bacteria that caused the cultures become negative. There were 2 samples reported with false negativity in TB antigen cocktail rapid ICT. One previous study using TB ICT kit detected false negativity in $6 \mathrm{M}$. tuberculosis isolates and genomic RD1-RD3 analysis showed mutation in all 6 strains..$^{15}$ The limitations of this study was no genetically confirmed $M$. tuberculosis strains to attribute to mutation occurring in the specific gene of M. tuberculosis isolates. Tuberculosis antigen cocktail rapid ICT

Table 3 Positivity Rate of TB Diagnostic Tests on Sputum and Cerebrospinal Fluid (CSF) Specimens

\begin{tabular}{lcccc}
\hline & \multicolumn{5}{c}{ Positivity Rate } \\
\cline { 2 - 5 } Diagnostic test & \multicolumn{2}{c}{ Sputum } & CSF & Number \\
\hline TB antigen cocktail & 58 & 40.8 & 16 & 39 \\
AFB microscopy & 59 & 39.6 & 2 & 5 \\
Cultured on Ogawa & 56 & 37.6 & 6 & 15 \\
\hline
\end{tabular}


sputum had a higher positivity rate $(40.8 \%)$ than $M$. tuberculosis microscopy $(39.6 \%)$ and M. tuberculosis culture (37.6\%).

Clinically and therapeutically differential identification of $M$. tuberculosis from MOTT is very important. Most of the Mycobacteriology laboratories identify $M$. tuberculosis using conventional biochemical tests. These tests are not labor sensitive and require special biosafety equipments. Biological, molecular, and immunological studies of $M$. tuberculosis complex have resulted in identification of different useful antigen, some of which are specific to $M$. tuberculosis complex. This study found none of TB antigen cocktail rapid ICT sputum positive in sample which had MOTT culture. It was suggested that TB antigen cocktail rapid ICT could distinguish $M$. tuberculosis complex infection from MOTT.

TB antigen cocktail rapid ICT from CSF specimens had a sensitivity of $83.3 \%$ and specificity of $68.5 \%$. Song et al. ${ }^{16}$ reported that the sensitivity TB antigen ESAT- 6 from CSF was $88 \%$ and the specificity was $92 \%$. In Kashyap et al. ${ }^{17}$ sensitivity of TB antigen ESAT6 was $80 \%$ and the specificity was $94 \%$. In both study, they detected antigen with ELISA method and used clinical diagnosis as a gold standard. In this study, culture was used as the gold standard; unfortunately culture is imperfect gold standard so the validity was lower than other study.

This study used the MODS culture as gold standard for CSF specimens. In previous study, it was found that MODS culture showed a high sensitivity and rapidity (compared with solid culture), simplicity, and potential low cost (compared with automated liquid systems) for diagnosis of TB meningitis in a hospital setting in Indonesia. ${ }^{18}$ Bacteriological confirmation of certain paucibacillary forms of TB, like TB meningitis, is problematic. In this study, $M$. tuberculosis microscopy and M. tuberculosis culture from CSF specimens had low positivity rate. The $M$. tuberculosis microscopy requires $5 \times 10^{3}-10^{4}$ organism $/ \mathrm{mL}$ and the culture requires $10^{1}-10^{2}$ viable organisms for positive result. In CSF, there were only about $10^{\circ}-10^{2}$ organism/mL. ${ }^{19}$ Tuberculosis antigen cocktail rapid ICT detect secreted antigen (ESAT-6, CFP-10 and MPT64) even if the bacteria was located in the cell or tissue. Therefore, the positivity rate of this assay higher (39\%) than other method (microscopy 5\%, culture $15 \%)$. Gain of the ICT method was a simple, rapid, easy, and high sensitivity makes the ICT a potential tool for rapid diagnosis of TB compared to other method.

In conclusions, the $\mathrm{TB}$ antigen cocktail rapid immunochromatography from sputum examination has good validity to diagnose pulmonary tuberculosis, and CSF has moderate validity to diagnose TB meningitis. Tuberculosis antigen cocktail rapid ICT sputum and CSF have a higher positivity rate than $M$. tuberculosis microscopy and M. tuberculosis culture.

\section{References}

1. Kunnath-Velayudhan K, Gennaro ML. Immunodiagnosis of tuberculosis: a dynamic view of biomarker discovery. Clin Microbiol Rev. 2011;24(4):792-805.

2. Fennelly KP, Morais CG, Hadad DJ, Vinhas $\mathrm{S}$, Dietze R, Palaci. M. The small membrane filter method of microscopy to diagnose pulmonary tuberculosis. J Clin Microbiol. 2012;50(6):2096-9.

3. Parkash O, Singh BP, Pai M. Regions of differences encoded antigens as targets for immunodiagnosis of tuberculosis in humans. Scan J Immunol. 2009;70(4):345-57.

4. Walzl G, Ronacher K, Hanekom W, Scriba TJ, Zumla A. Immunological biomarkers of tuberculosis. Nat Rev Immunol. 2011;11(5):343-54.

5. Mahairas GG, Sabo PJ, Hickey MJ, Singh DC,

Stover CK. Molecular analysis of genetic differences between Mycobacterium bovis BCG and virulent M. bovis. J Bacteriol. 1996;178(5):1274-82.

6. Ganguly N, Siddiqui I, Sharma P. Role of M. tuberculosis RD-1 region encoded secretory proteins in protective response and virulence. Tuberculosis. 2008;88(6):510-7.

7. Renshaw PS, Lightbody KL, Veverka V, Muskett FW, Kelly G, Frenkiel TA, et al. Structure and function of the complex formed by the tuberculosis virulence factors CFP-10 and ESAT-6. EMBO J. 2005;24(14):2491-8.

8. Lightbody KL, Ilghari D, Waters LC, Carey G, Bailey MA, Williamson RA, et al. Molecular features governing the stability and specificity of functional complex formation by mycobacterium tuberculosis CFP- 
10/ESAT-6 family proteins. J Biol Chem. 2008;283(25):17681-90.

9. Bekmurzayeva A, Sypabekova M, Kanayeva D. Tuberculosis diagnosis using immunodominant, secreted antigens of Mycobacterium tuberculosis. Tuberculosis 2013;93(4):381-8.

10. de Steenwinkel JE, de Knegt GJ, ten Kate MT, Verbrugh HA, Hernandez-Pando R, Leenen PJ, et al. Relapse of tuberculosis versus primary tuberculosis; course, pathogenesis and therapy in mice. Tuberculosis. 2013;93(2):213-21.

11. Yancey D. Tuberculosis. 241 First Avenue North Minneapolis, MN 55401 U.S.A.: Twenty-First Century Books A division of Lerner Publishing Group, Inc.; 2008.

12. Marais S, Thwaites G, Schoeman JF, Török ME, Misra UK, Prasad K, et al. Tuberculous meningitis: a uniform case definition for use in clinical research. Lancet Infect Dis. 2010;10(11):803-12

13. WHO. Global tuberculosis report 2012. Geneva, Switzerland: WHO; 2012.

14. Shen G, Chiou CS, Hu ST, Wu KM, Chen JH. Rapid identification of the mycobacterium tuberculosis complex by combining the esat6/cfp-10 immunochromatographic assay and smear morphology. J Clin Microbiol. 2011;49(3):902-7.
15. Ngamlert K1, Sinthuwattanawibool C, McCarthy KD, Sohn H, Starks A, Kanjanamongkolsiri P, et al. Diagnostic performance and costs of Capilia TB for Mycobacterium tuberculosis complex identification from broth-based culture in Bangkok, Thailand. Trop Med Int Health. 2009;23;14(7):748-53.

16. Song F, Sun X, Wang X, Nai Y, Liu Z. Early diagnosis of tuberculous meningitis by an indirect ELISA protocol based on the detection of the antigen ESAT-6 in cerebrospinal fluid. Ir J Med Sci. 2014;183(1):85-8.

17. Kashyap RS, Ramteke SS, Morey SH, Purohit HJ, Taori GM, Daginawala HF. Diagnostic value of early secreted antigenic target- 6 for the diagnosis of tuberculous meningitis patients. Infection. 2009;37(6):508-13.

18. Chaidir L, Annisa J, Dian S, Moore DAJ, Muhsinin S, Parwati I, et al. MODS culture for primary diagnosis of tuberculous meningitis and hiv-associated pulmonary tuberculosis in indonesia. Int J Trop Dis Health. 2013;3(4):34654.

19. Bhigjee I, Padayachee R, Paruk H, HallwirthPillay K, Marais S, Connoly C. Diagnosis of tuberculous meningitis: clinical and laboratory parameters. Int J Infect Dis. 2007;11(4):34854. 